\title{
Silicon on GaN(0001) and (000I) surfaces
}

C. D. Lee, R. M. Feenstra, A. L. Rosa, J. Neugebauer, and J. E. Northrup

Citation: Journal of Vacuum Science \& Technology B: Microelectronics and Nanometer Structures Processing, Measurement, and Phenomena 19, 1619 (2001); doi: 10.1116/1.1383074

View online: https://doi.org/10.1116/1.1383074

View Table of Contents: https://avs.scitation.org/toc/jvn/19/4

Published by the American Institute of Physics

\section{ARTICLES YOU MAY BE INTERESTED IN}

Reconstructions of GaN(0001) and (0001) surfaces: Ga-rich metallic structures Journal of Vacuum Science \& Technology B: Microelectronics and Nanometer Structures Processing, Measurement, and Phenomena 16, 2242 (1998); https://doi.org/10.1116/1.590156

Adsorption and incorporation of silicon at $\mathrm{GaN}(0001)$ surfaces

Applied Physics Letters 80, 2008 (2002); https://doi.org/10.1063/1.1452785

Determination of wurtzite GaN lattice polarity based on surface reconstruction Applied Physics Letters 72, 2114 (1998); https://doi.org/10.1063/1.121293

First-principles calculations for defects and impurities: Applications to III-nitrides Journal of Applied Physics 95, 3851 (2004); https://doi.org/10.1063/1.1682673

Band parameters for nitrogen-containing semiconductors Journal of Applied Physics 94, 3675 (2003); https://doi.org/10.1063/1.1600519

Morphology and surface reconstructions of $\mathrm{GaN}(1 \mathrm{I} 00)$ surfaces Applied Physics Letters 82, 1793 (2003); https://doi.org/10.1063/1.1560558 


\title{
Silicon on GaN(0001) and (0001) surfaces
}

\author{
C. D. Lee and R. M. Feenstra ${ }^{a)}$ \\ Department of Physics, Carnegie Mellon University, Pittsburgh, Pennsylvania 15213 \\ A. L. Rosa and J. Neugebauer \\ Fritz-Haber-Institut der Max-Planck-Gesellschaft, Faradayweg 4-6, D-14195 Berlin, Germany \\ J. E. Northrup \\ Xerox Palo Alto Research Center, 3333 Coyote Hill Road, Palo Alto, California 94304
}

(Received 9 January 2001; accepted 9 May 2001)

\begin{abstract}
Surface reconstructions and adatom kinetics of silicon on $\mathrm{GaN}(0001)$ and $(000 \overline{1})$ surfaces are studied by scanning tunneling microscopy, electron diffraction, and first-principles calculations. For silicon coverage near 0.5 monolayer, a $2 \times 2$ structure is observed, and is interpreted in terms of a model consisting of a $\mathrm{Ga}$ adatom on a monolayer of $3 \mathrm{Ga}+1 \mathrm{Si}$ and a $\mathrm{Si}_{\mathrm{Ga}}$ atom in the third layer. For higher silicon coverage, disordered $2 \times 2$ domains and " $1 \times 1$ ", domains are found to coexist. After annealing above $300^{\circ} \mathrm{C}$ the " $1 \times 1$ " regions become dominant and a $4 \times 4$ structure is seen near step edges. It is concluded that the silicon adatoms tend to reside in subsurface sites on the Ga-polar surface. Surface morphology in the presence of $\mathrm{Si}$ is smooth for the (0001) surface but rough for the $(000 \overline{1})$ surface. This difference is attributed to the presence of multiple Ga surface layers in the former case, which enhance surface diffusivities. (C) 2001 American Vacuum Society.
\end{abstract}

[DOI: $10.1116 / 1.1383074]$

\section{INTRODUCTION}

Silicon is commonly used as an $n$-type dopant in GaN. As in past studies of other doping systems, ${ }^{1-4}$ aspects of the surface science can determine limits on the incorporation efficiency and structural quality of the resulting films. Furthermore, it has been shown that silicon has a strong effect on the surface morphology of GaN films: small amounts of silicon on GaN modify the growth mode from step-flow to three-dimensional giving rise to the formation of small islands in metalorganic chemical vapor deposition (MOCVD) and gas source molecular beam epitaxy (MBE) ${ }^{5,6}$ The role of silicon adatoms in this morphological transition is, however, unclear. It is therefore important to study the details of $\mathrm{Si}$ incorporation and $\mathrm{Si}$-induced reconstruction of $\mathrm{GaN}$ surfaces.

In this study we investigate the effect of silicon exposure for both $\mathrm{GaN}(0001)$ and $(000 \overline{1})$ surfaces. Prior studies of the reconstructions of these surfaces, in the absence of $\mathrm{Si}$, have been reported. ${ }^{7,8}$ Both surfaces exhibit a number of reconstructions, depending on the surface stoichiometry. ${ }^{9,10}$ For the $\mathrm{GaN}(000 \overline{1})$ surface, or $\mathrm{N}$ face, the $\mathrm{Ga}$ atoms on the surface form a $1 \times 1$ structure with a Ga-Ga separation of $3.19 \AA$ A. By depositing submonolayer quantities of $\mathrm{Ga}$ onto this $1 \times 1$ surface additional reconstructions, with $3 \times 3,6$ $\times 6$, and $c(6 \times 12)$ symmetries, are produced. ${ }^{11,12}$ On the other hand, for the GaN (0001) surface or Ga face, several structures have been observed under Ga-poor conditions including $2 \times 2,5 \times 5$, and $6 \times 4$ reconstruction. ${ }^{13}$ Under Garich conditions it is found that there are about 2 monolayers $\left(1 \mathrm{ML}=1.14 \times 10^{15}\right.$ atoms $\left./ \mathrm{cm}^{2}\right)$ of $\mathrm{Ga}$ on top of the Gaterminated bilayer, ${ }^{14,15}$ with these Ga layers assuming some-

${ }^{a)}$ Electronic mail: feenstra@andrew.cmu.edu thing close to their bulk spacing so that they form an incommensurate structure on the surface. This structure is denoted by " $1 \times 1$ " (in quotation marks) or pseudo- $1 \times 1$, and it is found to play an important role in surface morphology of MBE-grown films. ${ }^{16}$

In this study, using scanning tunneling microscopy (STM) and reflection high-energy electron diffraction (RHEED), we observe a number of surface reconstructions produced by $\mathrm{Si}$ on GaN. The approximate Si coverage for these reconstructions is determined by Auger electron spectroscopy (AES). First-principles calculations are used to test various possible models for the reconstructions, focusing on the (0001) surface. We find that $\mathrm{Si}$ atoms strongly prefer to occupy subsurface sites, where they can form a maximum number of bonds to nitrogen. In fact, the calculations indicate that all of the Si-induced reconstructions of the $\mathrm{GaN}$ surface are unstable with respect to formation of $\mathrm{Si}_{3} \mathrm{~N}_{4}$. Experimentally we believe that $\mathrm{Si}_{3} \mathrm{~N}_{4}$ does not occur on our surfaces, thus indicating that the reconstructions which we have formed are all metastable. Another aspect of the experiments concerns the effect of silicon on the growth kinetics. We find that Si exposure on the (0001) surface maintains a smooth surface morphology, whereas Si exposure on the $(000 \overline{1})$ surface leads to a rough morphology. This difference is interpreted in terms of the presence of multiple $\mathrm{Ga}$ adlayers on the (0001) surface and their tendency to reduce surface diffusion barriers thereby promoting smooth growth.

\section{EXPERIMENT}

The experiments were performed in a combined MBE/ surface analysis system. Activated nitrogen is supplied by an SVTA radio frequency-plasma source, and effusion cells are used for $\mathrm{Ga}$ and various dopants. In particular, we use a 
mini electron source for silicon. The silicon beam flux is controlled by the beam current between the filament and silicon slug. Ga and Si beam fluxes were calibrated with a crystal thickness monitor, and the active $\mathrm{N}$ flux was calibrated by defining the $\mathrm{Ga} / \mathrm{N}$ flux ratio to be unity at the point where a transition between streaky and spotty behavior occurs in the RHEED pattern. ${ }^{17,18}$

In order to obtain the $\mathrm{GaN}(000 \overline{1})$ surface, $\mathrm{GaN}$ growth is initiated directly on sapphire substrates, with pregrowth nitridation of the substrate performed at $900{ }^{\circ} \mathrm{C}$ and using a low-temperature $\mathrm{GaN}$ buffer layer grown at $300^{\circ} \mathrm{C},{ }^{11}$ the film is found to be $\mathrm{N}$ polar (surface is $\mathrm{N}$ face). On the other hand, we use an MOCVD-grown GaN/sapphire film as an atomic-scale template for the Ga-polar films (surface is Ga face). In order to study the surface reconstructions using STM, it is necessary to dope the film with silicon (the precise doping concentration is not known, although the film resistivity is significantly changed by the Si doping). The doping was stopped shortly before terminating the film growth, and a 10-nm-thick undoped GaN layer was grown, on top of which the various $\mathrm{GaN}$ surface reconstructions were prepared. Silicon exposure was performed on these reconstructed surfaces at $300-350{ }^{\circ} \mathrm{C}$. At higher temperatures the $\mathrm{Si}$ induced reconstructions disappear. In fact, during growth, under silicon exposure, the surface does not show any reconstruction except $1 \times 1$.

Samples ready for investigation are transferred into the adjoining analysis chamber which includes STM and AES. The amount of silicon on the surface is determined from the silicon/nitrogen and silicon/gallium peak-peak intensity ratio as measured by the AES with an incident electron energy of $3 \mathrm{keV}$. By using sensitivity factors obtained from Ref. 19 and electron mean free path from Ref. 20, the amount of silicon is evaluated by averaging results from the ratios of $\mathrm{Si}$ $L M M(92 \mathrm{eV})$ to both $\mathrm{Ga} K L L(1070 \mathrm{eV})$ and $\mathrm{N}$ KLL (379 $\mathrm{eV})$ Auger lines. STM images were acquired with a constant tunnel current of $0.075 \mathrm{nA}$, and at various negative sample voltages specified later. Imaging at positive sample voltage of the $2 \times 2$ arrangement was unsuccessful to date, due either to some surface electronic property of this structure or to limited conductivity of the GaN films.

\section{EXPERIMENTAL RESULTS AND DISCUSSION}

\section{A. Low Si coverage: $2 \times 2$ reconstruction of GaN(0001)}

Most of our experimental studies have been carried out on the (0001) surface. If we deposit $\mathrm{Si}$ on a Ga-rich (0001) surface, displaying a " $1 \times 1$ " reconstruction, no change in the surface structure is observed by RHEED. The Si appears not to have modified the surface structure, as discussed in more detail later. If, alternatively, we deposit $\mathrm{Si}$ on an (0001) surface displaying a $5 \times 5$ reconstruction, a Si-induced 2 $\times 2$ reconstruction results. Figure 1(a) shows a STM image of neighboring areas of the $2 \times 2$ and $5 \times 5$ reconstructions. The initial $5 \times 5$ reconstruction was prepared by carefully adjusting the Ga flux during film growth such that the
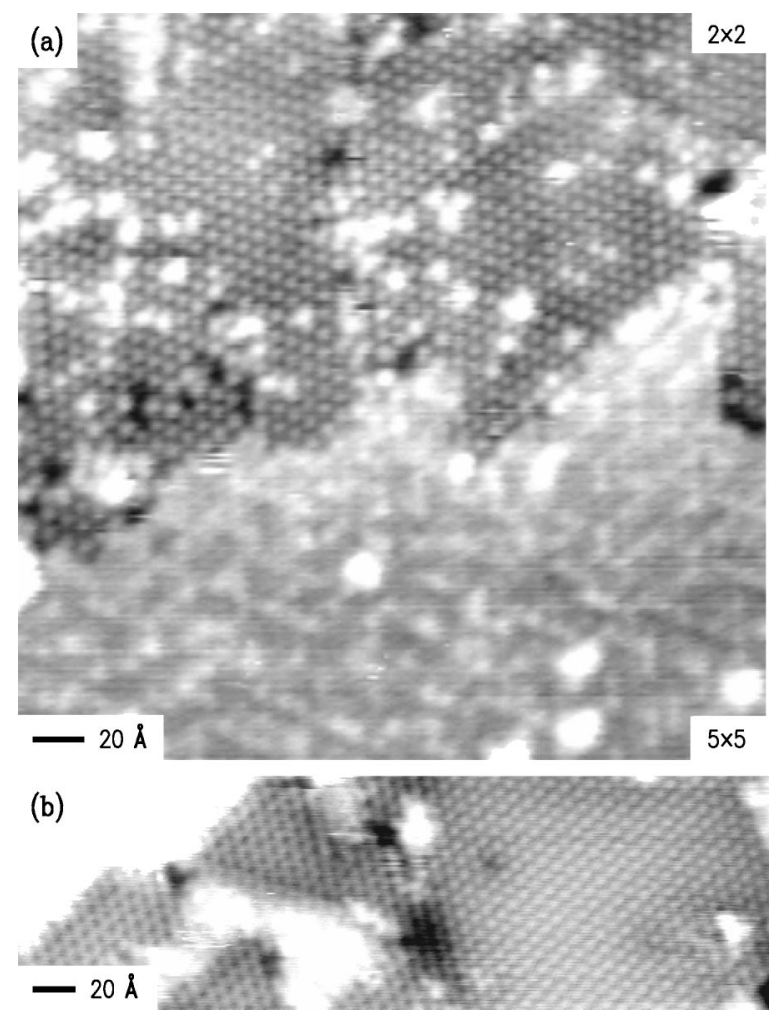

FIG. 1. STM images of GaN(0001) surface exposed to $\sim 0.5 \mathrm{ML}$ of silicon. (a) Surface region showing Si-induced $2 \times 2$ reconstruction and the $5 \times 5$ reconstruction of the bare surface. (b) Two different types of domains (seen on the left and right sides of the image) of the $2 \times 2$ structures. Images were acquired with sample bias voltages of -2.5 and $-2.0 \mathrm{~V}$, respectively, and are shown with gray-scale ranges of 1.3 and $1.0 \AA$, respectively.

RHEED showed a very faint $5 \times 5$ reconstruction. As the sample cooled this faint $5 \times 5$ become bright and clear over the entire surface area. At room temperature the sample does not show any other reconstructions except $5 \times 5$ in RHEED, which is consistent with STM observation. Silicon exposure was performed at a temperature near $300^{\circ} \mathrm{C}$. With sufficient silicon exposure a $2 \times 2$ pattern appears gradually. We note that the temperature window for formation of the $2 \times 2$ reconstruction is quite narrow. With increasing substrate temperature the $2 \times 2$ disappears after it has formed, implying that the $2 \times 2$ structure is metastable.

One interesting feature of the STM images is that two different domains of the $\mathrm{Si}$-induced $2 \times 2$ reconstructions by silicon are observed, as shown in Fig. 1(b). These domains might be caused of two different locations of the third layer $\mathrm{Si}$ atoms in this structure (which has only a very small effect on the total energy of the structure), as discussed in Sec. IV later.

We have performed AES on a $2 \times 2$ surface with a saturated $2 \times 2$ diffraction pattern, to estimate the concentration of adsorbed silicon atoms. Assuming that all the $\mathrm{Si}$ is in an adlayer on the surface we estimate a coverage of about 0.35 ML, with an estimated uncertainty in the analysis of $\pm 50 \%$. This coverage estimate changes, however, if we use a different model for position of the Si atoms. In particular, using the model of Sec. IV in which the Si is in the first layer (rest 

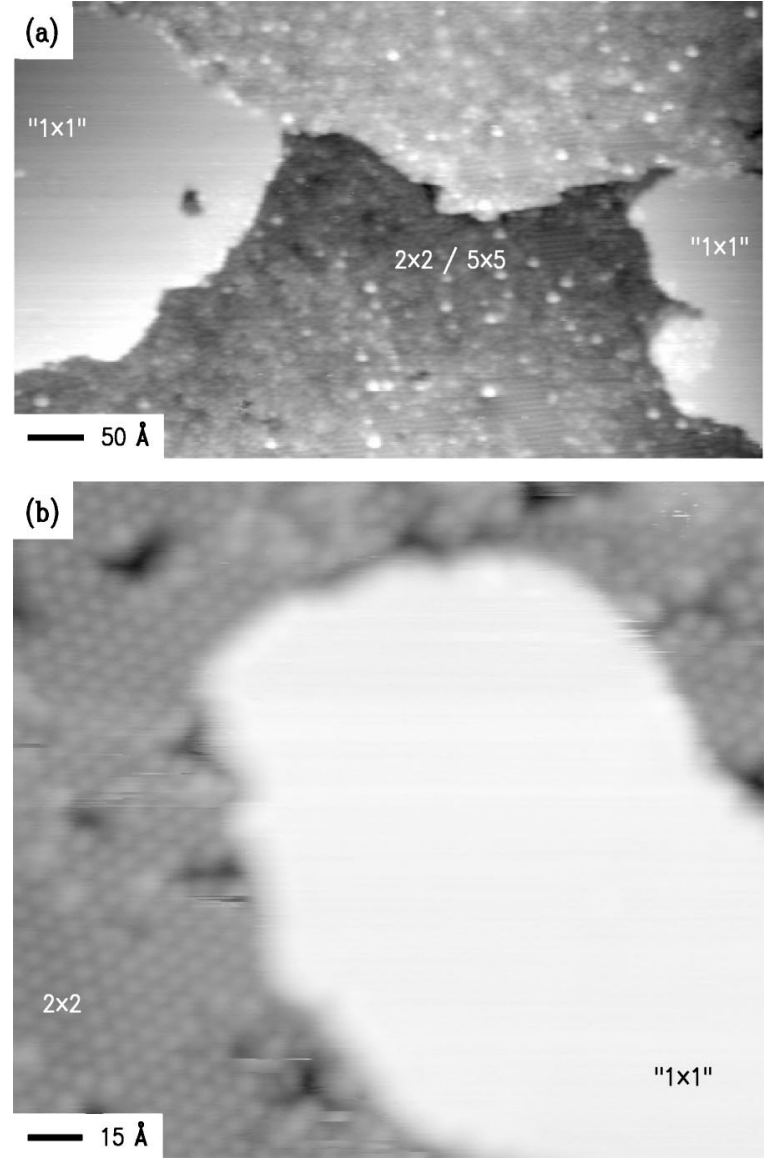

FIG. 2. STM image of $\mathrm{GaN}(0001)$ surface following $\sim 0.5$ ML silicon exposure. (a) Surface displaying regions of mixed $2 \times 2$ and $5 \times 5$ reconstructions, together with islands of " $1 \times 1$ " structures. (b) High resolution view of " $1 \times 1$ " island surrounded by $2 \times 2$ structure. Images were acquired with sample bias voltages of -2.0 and $-2.5 \mathrm{~V}$, respectively, and are shown with gray-scale ranges of 4.9 and $5.3 \AA$, respectively [image (b) is shown with a slightly nonlinear gray scale, to enhance the contrast in the $2 \times 2$ structure].

atom) and third layer below a Ga adatom layer, the computed Si Auger intensity is reduced due to the subsurface position of the third layer Si atoms. Using that structural model we estimate a Si coverage of 0.63 ML, assuming equal occupation of $\mathrm{Si}$ in the first and third layers. For the surface shown in Figs. 1 and 2, consisting mainly of $2 \times 2$ structure, we roughly estimate its $\mathrm{Si}$ coverage to be $\sim 0.5 \mathrm{ML}$. Silicon coverages for other surfaces presented below are estimated by scaling the exposure time of the Si compared to that of the surface of Figs. 1 and 2.

For surfaces prepared in the manner described earlier, we often observe by STM regions of both $2 \times 2$ and $5 \times 5$ reconstruction. The $5 \times 5$ has appearance (at both positive and negative sample voltages) which is identical to that seen on the bare surface, ${ }^{13}$ and we thus attribute its presence simply to incomplete Si coverage. On such $2 \times 2 / 5 \times 5$ surfaces we also invariably observe regions of the " $1 \times 1$ " reconstruction, as shown in Fig. 2. Figure 2(a) shows four adjacent terraces containing " $1 \times 1$ ', $5 \times 5$, and $2 \times 2$ reconstructions. The central part of the image, with two terraces separated by a bilayer-high step, consists of $2 \times 2$ and $5 \times 5$ struc- tures, with some of the $2 \times 2$ arrangement occurring in a fairly well-ordered, corrugated row-like structure over large areas. The terraces seen on the right and left sides of the image consist of " $1 \times 1$ " reconstruction. An interesting feature of Fig. 2(b) is that the " $1 \times 1$ " structure apparently does not react with silicon adatoms. Figure 2(b) shows an expanded view of a small island of " $1 \times 1$ " structure, surrounded by $2 \times 2$ reconstruction.

For filled state images, the " $1 \times 1$ " is found to be 2.54 $\pm 0.05 \AA$ higher than the $2 \times 2$ structure, and the $5 \times 5$ structure is found to be $0.62 \pm 0.05 \AA$ higher than the $2 \times 2$ structure. The height difference between " $1 \times 1$ " and $5 \times 5$ is found to be $1.92 \pm 0.05 \AA$ which is identical to the result obtained from the data of Ref. 13. It is important to note that the $2 \times 2$ structure is observed to be significantly lower than the $5 \times 5$ structure. Our model for the $2 \times 2$ structure, presented later, contains $\mathrm{Ga}$ adatoms (with $\mathrm{Si}$ rest atoms and subsurface atoms), and the somewhat tentative model for the $5 \times 5$ structure also contains $\mathrm{Ga}$ adatoms (and $\mathrm{N}$ adatoms). ${ }^{13}$ The observed height difference between the $2 \times 2$ and $5 \times 5$ structures must be accounted for in any future, refined structural modeling.

While STM images of the " $1 \times 1$ " typically appear featureless (except at very high resolution), it is not uncommon to observe small domains of a different reconstruction near the edges of the " $1 \times 1$ " domains, as seen in the " $1 \times 1$ " region on the right-hand side of Fig. 2(a). Similar structures on top of " $1 \times 1$ " regions have been previously reported. ${ }^{13}$

\section{B. High Si coverage: $4 \times 4$ reconstruction of GaN(0001)}

When additional $\mathrm{Si}$, above $\sim 0.5 \mathrm{ML}$, is deposited on the surface, the $1 / 2$ order diffraction lines seen in RHEED become dim. The resulting surface appears in STM to be disordered, as shown in Fig. 3. Small domains of well-ordered $2 \times 2$ reconstruction are seen on the surface, as well as numerous small islands with " $1 \times 1$ " reconstruction. Relative to the initial Si exposure, the density of " $1 \times 1$ " domains increases, implying that the silicon adatoms substitute for the $\mathrm{Ga}$ atoms and the resulting ejected $\mathrm{Ga}$ tends to form metallic adlayers on the surface. If we anneal this surface briefly at $350^{\circ} \mathrm{C}$, and cool to room temperature, the RHEED pattern shows a mixed " $1 \times 1$ " and $2 \times 2$ pattern, consistent with the STM observation.

Upon continuing the silicon exposure up to $\sim 1 \mathrm{ML}$ at $300^{\circ} \mathrm{C}$, the $2 \times 2$ diffraction pattern becomes weak and a 4 $\times 4$ pattern appears. The RHEED pattern is diffuse, indicating some surface disorder. In addition to the $4 \times 4$, the RHEED pattern shows a weak " $1 \times 1$ " pattern at room temperature. (We note that it is difficult to distinguish the 4 $\times 4$ pattern from a pattern formed by overlapping " $1 \times 1$ " and $2 \times 2$, but close inspection of the RHEED patterns does indicate that the $4 \times 4$ is real and distinct.) After careful annealing at around $350^{\circ} \mathrm{C}$ for $2 \mathrm{~min}$, the RHEED pattern shows a clear a $4 \times 4$ reconstruction. A large scale STM image for this sample shows in Fig. 4(a), and a detailed view of the $4 \times 4$ is shown in Fig. 4(b). As seen there, the feature- 


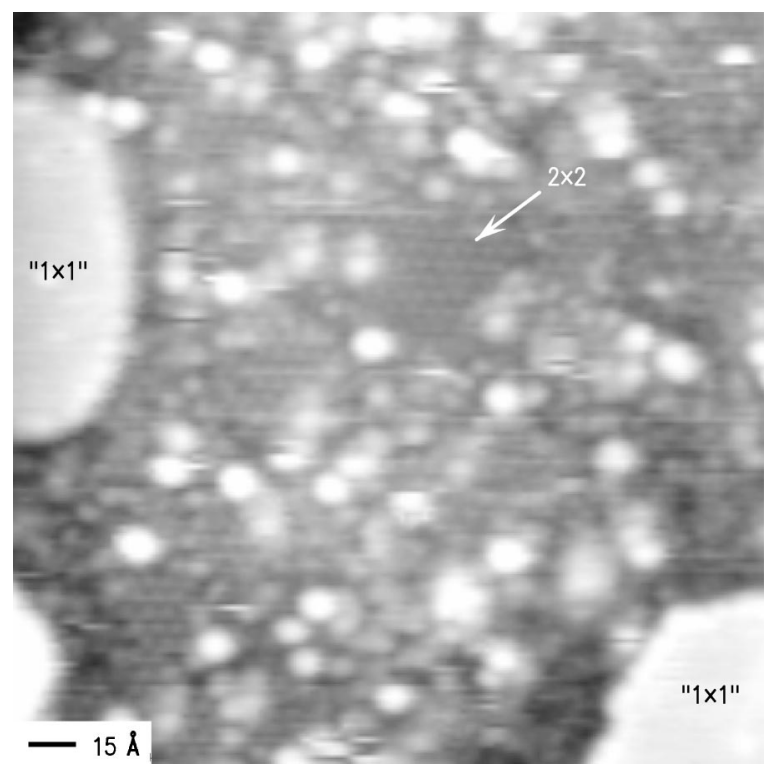

FIG. 3. STM image of $\mathrm{GaN}(0001)$ surface following $\sim 0.75 \mathrm{ML}$ silicon exposure. A region of adatom covered $2 \times 2$ structure is indicated. The regions labeled " $1 \times 1$ ", are domains of " $1 \times 1$ " reconstruction which are saturated in the gray-scale image. The smaller white clusters have heights of nearly $2.4 \AA$, and thus may be the initial stages of " $1 \times 1$ " domains. The image was acquired with a sample bias voltage of $-3.0 \mathrm{~V}$ and is shown with a gray-scale range of $3.0 \AA$.
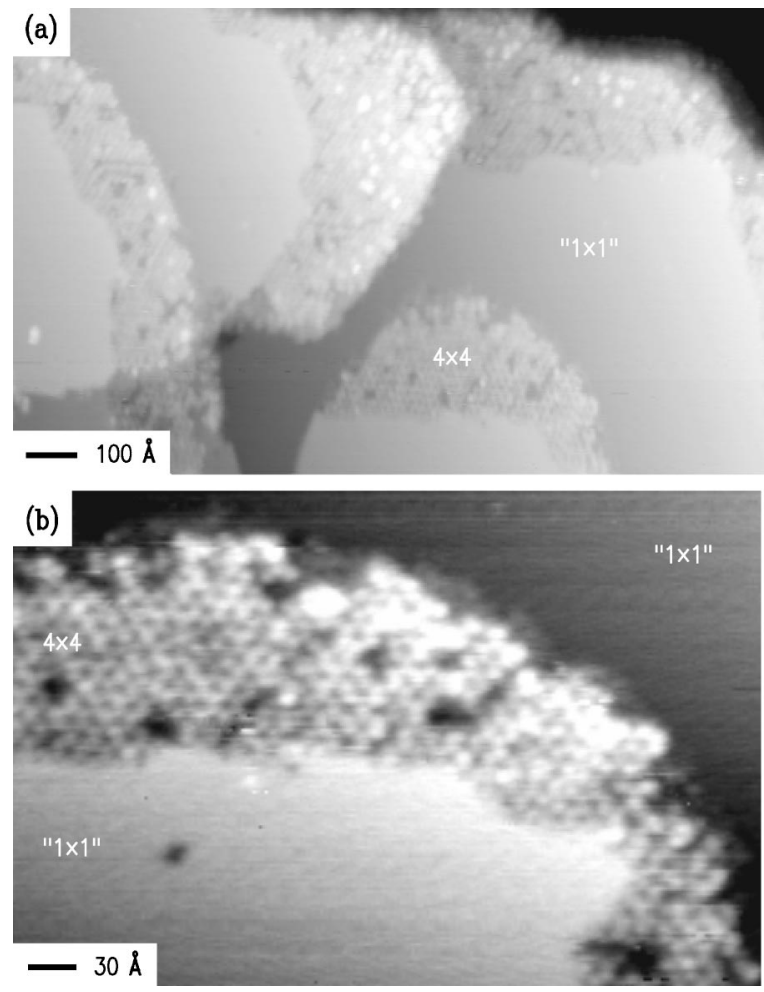

FIG. 4. STM image of GaN(0001) surface following $\sim 1$ ML silicon exposure. (a) Large scale image displaying terraces of " $1 \times 1$ " reconstruction with $4 \times 4$ structure seen at the terrace edges. (b) High resolution view of $4 \times 4$ structure near a terrace edge. Images were both acquired with a sample bias voltages of $+2.0 \mathrm{~V}$, and are shown with gray-scale ranges of 13 and 2.1 $\AA$, respectively. (a)

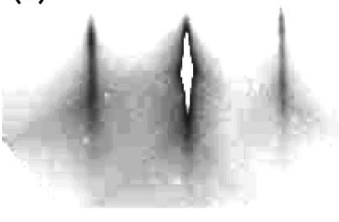

(b)

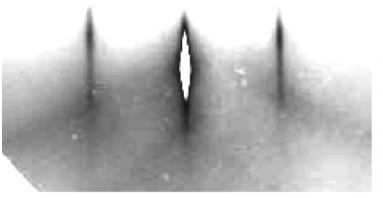

(c)

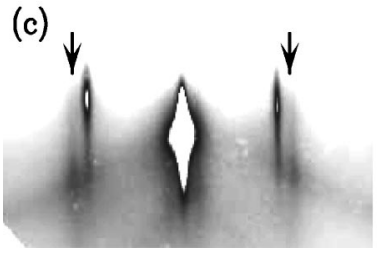

(d)

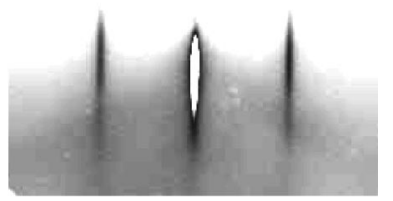

(e)

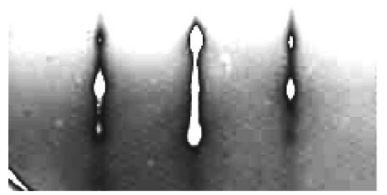

(f)

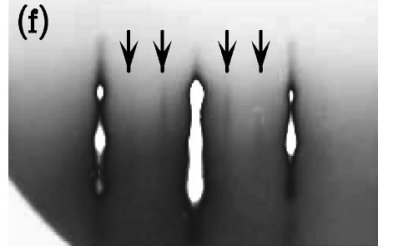

FIG. 5. RHEED images of GaN surface, along [11 $\overline{2} 0]$ azimuth. (a)-(c) (0001) polarity: (a) during growth in absence of $\mathrm{Si}$, (b) during growth in presence of $\mathrm{Si}$, and (c) at room temperature, following the growth in the presence of $\mathrm{Si}$. Arrows in (c) show the sidebands of the " $1 \times 1$ "' reconstruc-

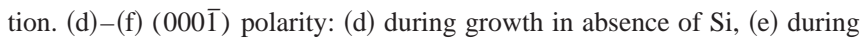
growth in presence of $\mathrm{Si}$, and (f) at room temperature, following the growth in the presence of Si. Arrows in (f) show weak 1/3-order fringes of the 3 $\times 3$ reconstruction.

less " $1 \times 1$ " region is dominant and the $4 \times 4$ region is seen only near step edges. With increasing anneal temperature, the $4 \times 4$ disappears completely, and at room temperature only the " $1 \times 1$ " reconstruction is be seen. This indicates that the whole surface is covered by the $\sim 2 \mathrm{ML} \mathrm{Ga}$ bilayer and the silicon atoms move to subsurface sites. Thus, based on these experimental observations we conclude that the silicon adatoms tend to reside in subsurface sites on the Gapolar surface.

\section{Effect of silicon on the smooth-to-rough transition}

As discussed earlier, MBE growth of $\mathrm{GaN}(0001)$ or $(000 \overline{1})$ displays a characteristic smooth-to-rough transition when the $\mathrm{Ga}$ to $\mathrm{N}$ flux ratio decreases below unity. We have observed here a dramatic difference in the smooth-to-rough behavior between the (0001) and (0001) faces in the presence of silicon. For both experiments the growth conditions were identical; in particular, the silicon beam flux was the same. Figure 5 shows the morphological evolution produced by the silicon incorporation. In the absence of silicon, the RHEED patterns of both polarities shows a streaky $1 \times 1$ reconstructions, implying the smooth surfaces proceed during the film growth [in Figs. 5(a) and 5(d)]. When the silicon exposure starts, the RHEED pattern of (0001) surface become slightly brighter but does not show any significant changes [Fig. 5(b)]. After termination of the film growth, the sample was cooled to room temperature. The (0001) surface then shows distinct sidebands on the high wavevector sides of the first-order streaks along the $[11 \overline{2} 0]$ azimuth [as indi- 

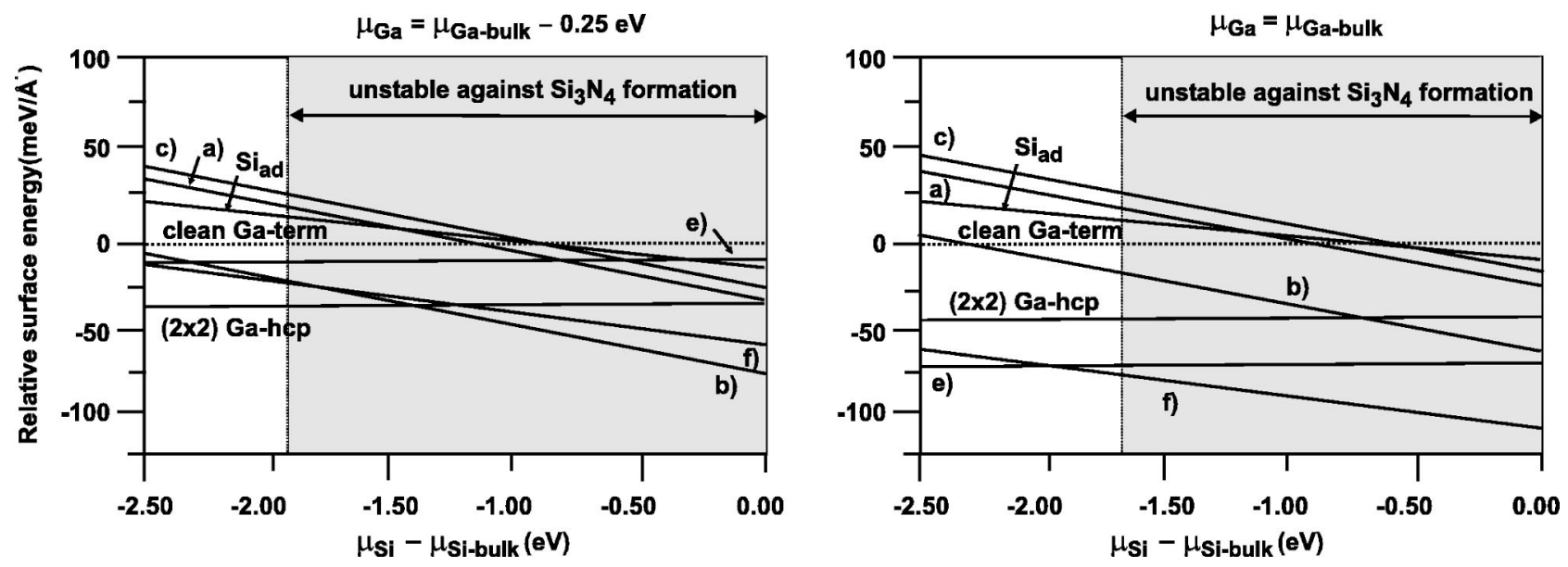

FIG. 6. Surface energies for the various reconstructions as shown in Fig. 7. Medium Ga-rich conditions (left side) and extreme Ga-rich conditions (right side) are shown. The labels (a)-(f) refer to the structures as shown in Fig. $7 . \mathrm{Si}_{\mathrm{ad}}$ marks a $2 \times 2$ reconstruction consisting of a single $\mathrm{Si}$ adatom on a Ga-terminated surface.

cated in Fig. 5(c) by arrows], indicative of the " $1 \times 1$ " reconstruction. This result demonstrates that during regular GaN(0001) film growth under Ga-rich conditions silicon incorporation does not affect the surface morphology-we believe that $\mathrm{Si}$ is still incorporated into the film, but it goes immediately into subsurface sites so that it does not affect the $\sim 2 \mathrm{ML}$ of $\mathrm{Ga}$ on the surface. This bilayer of excess $\mathrm{Ga}$ promotes surface diffusion by reducing diffusion barriers, ${ }^{16}$ and since the Si does not disturb this bilayer it thus does not affect the surface kinetics.

In contrast to the earlier results for the Ga face, Si deposition during growth on the $(000 \overline{1})$ surface leads immediately to a spotty RHEED pattern [Fig. 5(e)], indicating the surface is roughened in the presence of silicon. When cooled down to room temperature this surface shows a weak, spotty $3 \times 3$ reconstruction. This $3 \times 3$ structure is the same as observed for $\mathrm{GaN}(000 \overline{1})$ growth in the absence of $\mathrm{Si}$. We interpret the $3 \times 3$ spots as arising from residual surface areas which have not been affected by the Si. Indeed, if we expose an initially $3 \times 3 \mathrm{GaN}(000 \overline{1})$ surface to $\mathrm{Si}$ at a temperature of $300-350{ }^{\circ} \mathrm{C}$, we find that the $3 \times 3$ structure immediately disappears (i.e., for $\mathrm{Si}$ exposure much less than $1 \mathrm{ML}$ ). We conclude that $\mathrm{Si}$ exposure of the $(000 \overline{1})$ surface affects both the surface reconstruction and the morphology. We speculate that the $\mathrm{Si}$ resides in sites in the surface layer (i.e., substituting for the terminated $\mathrm{Ga}$ layer on the surface), and these $\mathrm{Si}$ atoms are not covered by excess Ga atoms. Thus, any enhancement in surface diffusivities produced by excess $\mathrm{Ga}$ is lost in this case.

Finally, we comment briefly on a reconstruction which we have observed on the $(000 \overline{1})$ surface upon $\mathrm{Si}$ exposure. When we expose the $c(6 \times 12)$ reconstruction to $\sim 1 \mathrm{ML}$ of $\mathrm{Si}$ at a temperature of $300-350{ }^{\circ} \mathrm{C}$, we observe in RHEED the appearance of a clear $7 \times 7$ reconstruction. We have verified this structure using low-energy electron diffraction also. Several attempts at STM characterization of this surface have been unsuccessful due to limited conductance of the sample.
Further work is needed to elucidate the atomic arrangement of this reconstruction.

\section{THEORY}

In order to identify the chemical nature of the Si-induced reconstructions we have performed first-principles total energy calculations for a variety of Si-terminated GaN surfaces. The $\mathrm{Si}$ coverage has been varied between $1 / 4$ and 2 monolayers (ML). The Si atoms have been placed on various high symmetry surface sites (face-centered-cubic, hexagonalclose-packed, on top) as well as on $\mathrm{N}$ and Ga substitutional sites on the surface and in the subsurface region. The calculations and results are discussed in detail elsewhere. ${ }^{21}$ Here we will focus on chemical trends and possible candidate structures for the $\mathrm{Si}$ induced $2 \times 2$ reconstruction.

Analyzing the energetics of all calculated surface structures we can derive a number of principles guiding the incorporation of Si: (i) Si substitutes always on a Ga site (incorporation on a $\mathrm{N}$ site is energetically highly unfavorable). (ii) $\mathrm{Si}$ attempts to maximize the number of $\mathrm{Si}-\mathrm{N}$ bonds. (iii) All surface structures containing $\mathrm{Si}$ are thermodynamically unstable against the formation of $\mathrm{Si}_{3} \mathrm{~N}_{4}$, except for very Garich conditions (see later).

Using these guiding principles we constructed various model structures to identify candidates for the Si induced 2 $\times 2$ reconstruction on $\mathrm{GaN}(0001)$. As a first set of structures we considered a $\mathrm{Si}$ adatom on a Ga-terminated surface where 0 or 1 of the $\mathrm{Ga}$ atoms in the Ga surface layer have been replaced by $\mathrm{Si}$ atoms. This corresponds to a $\mathrm{Si}$ coverage of $\Theta_{\mathrm{Si}}=1 / 4$ and $1 / 2 \mathrm{ML}$, respectively. The calculated surface energies for these structures are shown on the left side of Fig. 6 as function of the Si chemical potential. For Fig. 6(a) we have assumed medium Ga-rich conditions $\left(\mu_{\mathrm{Ga}}=\mu_{\mathrm{Ga}-\text { bulk }}\right.$ $-0.25 \mathrm{eV})$. At those conditions, and in the absence of $\mathrm{Si}$, the $5 \times 5$ reconstruction of bare $\mathrm{GaN}(0001)$ is stable. ${ }^{13}$

As can be seen in Fig. 6, the energetically most stable reconstruction consists of two $\mathrm{Si}$ atoms [a $\mathrm{Si}$ adatom and a $\mathrm{Si}$ 


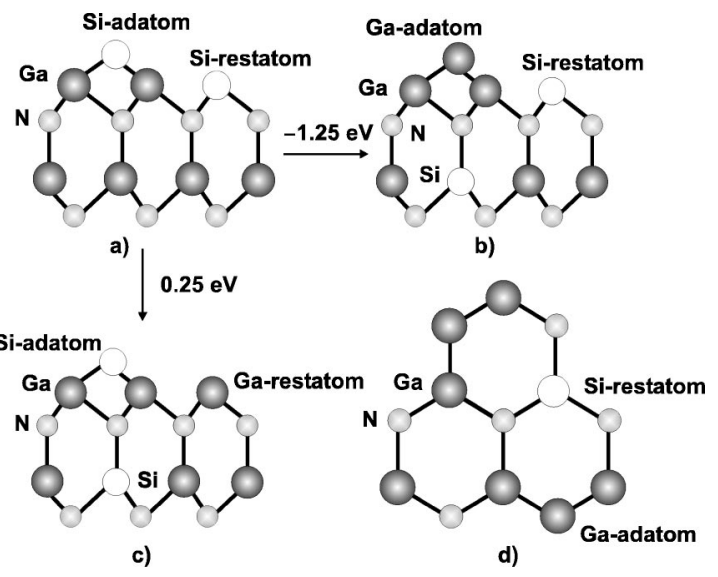

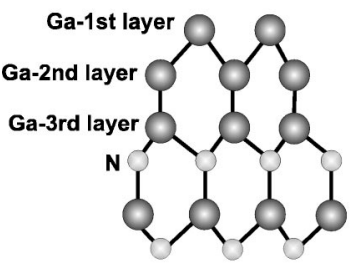

e)

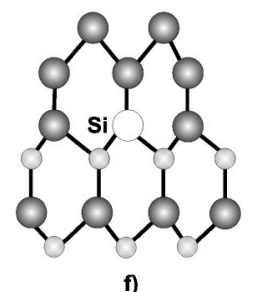

FIG. 7. Schematic geometry of the bare and Si-induced GAN(0001) surface reconstructions. (a) $\mathrm{Si}$ adatom $+\mathrm{Si}$ restatom structure, (b) the same structure but exchanging the $\mathrm{Si}$ adatom with a third layer $\mathrm{Ga}$ atom, (c) the same structure as in (a) but exchanging the Si restatom with a third layer Ga atom. The top view of (b) is shown in (d). Also indicated are the energy differences per $2 \times 2$ cell for these structures. Positive numbers correspond to an exothermic reaction. (e) and (f) show a " $1 \times 1$ " Ga bilayer structure without and with a $\mathrm{Si}$ atom in the third layer, respectively.

restatom; see Fig. 7(a)]. In a second step we checked whether this structure is stable against exchanging the Si adatom and rest atom with $\mathrm{Ga}$ atoms in the third layer. This exchange is motivated by the guiding principles mentioned earlier, namely, that $\mathrm{Si}$ atoms on $\mathrm{GaN}$ prefer the configuration with the highest number of $\mathrm{Si}-\mathrm{N}$ bonds. Indeed, among this set of structures we find a reconstruction with a particularly low energy. This structure consists of a $\mathrm{Ga}$ adatom and a $\mathrm{Si}$ atom in the third layer [see Figs. 7(b) and 7(d)]. The structure is $1.25 \mathrm{eV} / 2 \times 2$ cell lower in energy than the original $2 \times 2$ adatom structure. An exchange of the restatom with a $\mathrm{Ga}$ atom in the third layer leads to a less stable surface which is $0.25 \mathrm{eV}$ higher in energy [Fig. 7(c)]. We therefore identify the $\mathrm{Si}$ induced $2 \times 2$ structure seen in STM with the model shown in Figs. 7(b) and 7(d). We note that this structure is the energetically most favorable one only in a region of chemical potentials which is intrinsically unstable against the formation of $\mathrm{Si}_{3} \mathrm{~N}_{4}$.

The large energy difference between the two structures shown in Figs. 7(a) and 7(b) implies that Si adatoms on the surface are highly unstable against incorporation in bulk $\mathrm{GaN}$. This effect will lead to a very efficient Si incorporation in bulk GaN. It is interesting to note that this effect is opposite to what has been found for other impurities ( $\mathrm{In}, \mathrm{O}, \mathrm{Mg}$ ) which all prefer configurations on the surface. This tendency of $\mathrm{Si}$ to easily incorporate in bulk $\mathrm{GaN}$ might also explain why no $\mathrm{Si}_{3} \mathrm{~N}_{4}$ is formed although all $\mathrm{Si}$ containing
$\mathrm{GaN}(0001)$ surfaces are thermodynamically unstable against its formation. The efficient incorporation of $\mathrm{Si}$ in bulk prevents an accumulation on the surface which eventually would lead to the formation of $\mathrm{Si}_{3} \mathrm{~N}_{4}$.

It is important to note that the reaction of $\mathrm{Si}$ with the bare $\mathrm{GaN}$ surface produces excess Ga atoms. For example, in the Si-induced $2 \times 2$ structure [Fig. 7(b)] two $\mathrm{Si}$ atoms replace two Ga atoms, i.e., in this reaction two excess Ga atoms per $2 \times 2$ cell are created. This mechanism drives the system towards more Ga-rich conditions (the Ga chemical potential increases). On the right side of Fig. 6 we have therefore plotted the surface energies for Ga-rich conditions, i.e., for the upper limit of the Ga-chemical potential $\left(\mu_{\mathrm{Ga}}\right.$ $=\mu_{\mathrm{Ga}-\text { bulk }}$ ). In the absence of $\mathrm{Si}$ (for $\mu_{\mathrm{Si}} \rightarrow-\infty$ ) the $\mathrm{Ga}$ bilayer [Fig. 7(e)] is the most stable bare GaN surface. At sufficiently high $\mathrm{Si}$ chemical potential $\left(\mu_{\mathrm{Si}}=\mu_{\mathrm{Si}-\text { bulk }}\right.$ $-2.0 \mathrm{eV})$ a modified Ga-bilayer structure becomes energetically favored where a $\mathrm{Si}$ atom replaces a $\mathrm{Ga}$ atom in the third layer [see Fig. 7(f)]. We note, that since the Si atom is covered by a metallic bilayer the structure will be in STM identical to the bare Ga-bilayer structure. It is important to note that the structure is stable against the formation of $\mathrm{Si}_{3} \mathrm{~N}_{4}$, in contrast to the $\mathrm{Si}$-induced $2 \times 2$ structures.

Based on these results we interpret the structural changes observed in STM as follows. If Si adsorbs on the bare $\mathrm{GaN}$ surface it kicks out surface Ga atoms and induces a $2 \times 2$ reconstruction. The excess $\mathrm{Ga}$ atoms cluster in islands and form a Ga bilayer with " $1 \times 1$ " structure stabilized by $\mathrm{Si}$ atoms in the third layer. With increasing Si coverage more and more excess $\mathrm{Ga}$ atoms are created-the area covered by the Ga-bilayer increases until eventually it covers the entire surface.

\section{CONCLUSIONS}

In summary, we have studied surface reconstructions and adatom kinetics of silicon on $\mathrm{GaN}(0001)$ and (0001) surfaces by scanning tunneling microscopy, electron diffraction, and first-principles calculations. In the low silicon coverage regime, with less than $\lesssim 0.5 \mathrm{ML}$ of silicon, a $2 \times 2$ structure is observed by the silicon exposure on a $5 \times 5-\mathrm{GaN}(0001)$ surface at $\sim 300{ }^{\circ} \mathrm{C}$. Based on the theoretical calculation we propose that the $2 \times 2$ surface is consisting of a $\mathrm{Ga}$ adatom on a monolayer of $3 \mathrm{Ga}+1 \mathrm{Si}$ and $\mathrm{Si}_{\mathrm{Ga}}$ atom in the third layer. With high silicon coverage of about $1 \mathrm{ML}$, a $4 \times 4$ structure appears together with disordered $2 \times 2$ domains and " $1 \times 1$ " domains. After annealing above $300{ }^{\circ} \mathrm{C}$ the " $1 \times 1$ ", regions become dominant and the $4 \times 4$ structure is seen only near step edges. Based on these experimental observations we conclude that the silicon adatoms tend to reside in subsurface sites on the Ga-polar surface, implying that siliconinduced reconstruction is metastable. Smooth growth morphology is found for this Ga-rich (0001) surface, consistent with that found in our prior studies in which multiple metal layers are present on the surface.

The effect of Si exposure during growth depends on the Ga coverage of the growth surface. Most MBE is performed in a Ga-rich regime with a double layer of Ga present on the 
surface. We observe in this case that Si does not modify the surface structure, which we take to signify that the Si atoms all go subsurface. In contrast, on other semiconductors surfaces such as $\mathrm{GaAs}(001), \mathrm{Si}$ is found to modify the surface reconstruction. ${ }^{1,2}$ This difference between $\mathrm{GaN}(0001)$ and $\mathrm{GaAs}(001)$ arises, we believe, from the presence of the metallic Ga bilayer on the former surface and its absence on the latter.

Recent work by Munkholm et al. using in situ X-ray scattering on $\mathrm{GaN}(0001)$ surfaces grown by MOCVD reveals that the presence of $\mathrm{Si}$ leads to a transformation in the growth mode from step-flow to layer-by-layer. ${ }^{4}$ This results differs from ours, in which we find for Ga-rich plasmaassisted MBE that the growth mode is always step flow (at least for coverages of Si up to $2 \mathrm{ML}$ ). We believe that the difference between the two cases may again be attributed to the absence of the Ga bilayer in the MOCVD case, although it should be noted that no detailed information is currently available on the surface stoichoimetry during MOCVD growth.

Finally, in contrast to our results for the (0001) surface, we find that $\mathrm{Si}$ exposure on the (0001) surface leads to a rough morphology. We again tentatively interpret this result as being due to the absence of multiple metallic $\mathrm{Ga}$ adlayers on this surface.

\section{ACKNOWLEDGMENTS}

The authors acknowledge the contributions of Dr. S. J. Son for providing high quality MOCVD-grown GaN wafers. This work was supported by the Office of Naval Research under Grant No. N00014-96-1-0214, monitored by Dr. C. Wood. J. N. and A. L. R. thank the German Research Society for financial support.

${ }^{1}$ M. D. Pashley, K. W. Haberern, and R. M. Feenstra, J. Vac. Sci. Technol. B 10, 1874 (1992).
${ }^{2}$ Z. M. Wang, L. Däweritz, P. Schützendübe, and K. H. Ploog, J. Vac. Sci. Technol. B 18, 2204 (2000).

${ }^{3}$ V. Ramachandran, R. M. Feenstra, W. L. Sarney, L. Salamanca-Riba, J. E. Northrup, L. T. Romano, and D. W. Greve, Appl. Phys. Lett. 75, 808 (1999).

${ }^{4}$ A. Munkholm et al., Appl. Phys. Lett. 77, 1626 (2000).

${ }^{5}$ S. Tanaka, S. Iwai, and Y. Aoyagi, Appl. Phys. Lett. 69, 4096 (1996).

${ }^{6}$ X.-Q. Shen, S. Tanaka, S. Iwai, and Y. Aoyagi, Appl. Phys. Lett. 72, 344 (1998).

${ }^{7}$ A. R. Smith, R. M. Feenstra, D. W. Greve, M.-S. Shih, M. Skowronski, J. Neugebauer, and J. E. Northrup, Appl. Phys. Lett. 72, 2114 (1998).

${ }^{8}$ A. R. Smith, V. Ramachandran, R. M. Feenstra, D. W. Greve, M.-S. Shin, M. Skowronski, J. Neugebauer, and J. E. Northrup, J. Vac. Sci. Technol. A 16, 1641 (1998).

${ }^{9}$ M. M. Sung, J. Ahn, V. Bykov, J. W. Rabalais, D. D. Koleske, and A. E. Wickenden, Phys. Rev. B 54, 14652 (1996); J. Ahn, M. M. Sung, J. W. Rabalais, D. D. Koleske, and A. E. Wickenden, J. Chem. Phys. 107, 9577 (1997).

${ }^{10}$ M. A. Khan, J. N. Kuznia, D. T. Olson, and R. Kaplan, J. Appl. Phys. 73, 3108 (1993).

${ }^{11}$ A. R. Smith, R. M. Feenstra, D. W. Greve, J. Neugebauer, and J. E. Northrup, Phys. Rev. Lett. 79, 3934 (1997).

${ }^{12}$ A. R. Smith, R. M. Feenstra, D. W. Greve, J. Neugebauer, and J. E. Northrup, Appl. Phys. A: Mater. Sci. Process. 66, S947 (1998).

${ }^{13}$ A. R. Smith, R. M. Feenstra, D. W. Greve, M.-S. Shin, M. Skowronski, J. Neugebauer, and J. E. Northrup, Surf. Sci. 423, 70 (1999).

${ }^{14}$ A. R. Smith, R. M. Feenstra, D. W. Greve, M.-S. Shin, M. Skowronski, J. Neugebauer, and J. Northrup, J. Vac. Sci. Technol. B 16, 2242 (1998).

${ }^{15}$ J. E. Northrup, J. Neugebauer, R. M. Feenstra, and A. R. Smith, Phys. Rev. B 61, 9932 (2000).

${ }^{16}$ H. Chen, R. M. Feenstra, J. E. Northrup, T. Zywietz, J. Neugebauer, and D. W. Greve, J. Vac. Sci. Technol. B 18, 2284 (2000).

${ }^{17}$ E. J. Tarsa, B. Heying, X. H. Wu, P. Fini, S. P. DenBaars, and J. S. Speck, J. Appl. Phys. 82, 5472 (1997).

${ }^{18}$ A. R. Smith et al., MRS Internet J. Nitride Semicond. Res. 3, 12 (1998).

${ }^{19}$ L. E. Davis, N. C. MacDonald, P. W. Palmberg, G. E. Riach, and R. E. Weber, Handbook of Auger Electron Spectroscopy (Physical Electronics Industries, Eden Prairie, MN, 1978).

${ }^{20}$ S. Mroczkowski and D. Lichtman, Surf. Sci. 131, 159 (1983).

${ }^{21}$ A. L. Rosa, J. Neugebauer, J. E. Northrup, C. D. Lee, and R. M. Feenstra (unpublished). 